\title{
Intracellular Localization of Foot-and-Mouth Disease Virus Transgene Expression in vivo and in vitro After Infection with Adenovirus Vaccine Constructs
}

Thomas G. Burrage ${ }^{1}$, Kelsey R. Hilton ${ }^{1,5}$, Edward J. Kramer ${ }^{2}$, Jonathan Arzt ${ }^{2}$, Jose Barrera $^{1}$, Chris Shutta ${ }^{1}$, Marvin J. Grubman ${ }^{2}$, Doug Brough ${ }^{3}$, Alfonso Clavijo ${ }^{4}$, David A. Brake $^{1}$ and John Neilan ${ }^{1}$

${ }^{1}$ Department of Homeland Security, Plum Island Animal Disease Center

${ }^{2}$ Agricultural Research Service, USDA, Plum Island Animal Disease Center

${ }^{3}$ GenVec, Inc, Gaithersburg, Maryland.

${ }^{4}$ National Centre for Foreign Animal Disease, Winnipeg, Manitoba, Canada

${ }^{5}$ Oak Ridge Institute for Science and Education, Oak Ridge, TN

A replication defective adenovirus platform (Ad5) has been successfully used to immunize livestock against foot-and-mouth disease [1]. Ultrastructural studies of Ad5 infected continuous cell lines reveal picornavirus-like replication complexes and a proliferation of single membrane bound vesicles [2]. In this study, we follow sub-cellular transgene expression in primary and continuous cell culture lines infected with Ad5platform constructs to FMDV isolates using monoclonal antibodies (Mabs F14 and F21) and confocal microscopy (Figs 1A-1C). We also demonstrate the $48 \mathrm{hr}$ post immunization distribution of FMDV transgene expression in the ipsilateral and contralateral draining lymph nodes (Figs 2A and 2B). To investigate the sub-cellular consequences of Ad5 construct infection we used conventional electron (Fig.3) and immunoelectron microscopy (Fig. 4) to demonstrate the proliferation of single membrane vesicles and the distribution of Mab 14 reactive material. Qualitative and quantitative ultrastructural changes also occur during expression of different constructs. These changes may be predictive of vaccine efficacy and are a potential model of in vivo expression events.

\section{References}

[1] Pena et al., Vaccine 23 (2008) 5689.

[2] Moraes et al., Vaccine 20 (2002) 1631.

Figures 1A-1C. Assembled confocal z-stacks of three FMDV Ad5-constructs incubated with Mab F14 and anti-mouse Alexa 488 in LFBK cells. Nuclei stained with Topro 3. Figure 2A. Assembled confocal z-stack of large mobile cells in draining lymph node incubated with FMDV Mab F14. Figure 2B. Assembled confocal z-stack of germinal center cells in ispsilateral draining lymph node with Mab F14 showing punctate staining. Figure 3. Electron micrograph of LFBK cell line infected with Ad5-FMDV vector showing proliferation of single membrane vesicles and replication like complexes. Figure 4. Immunoelectron micrograph of LFBK cell line infected with Ad5-FMDV vector incubated in situ with Mab 14 and gold-enhanced immuno-gold. No heavy metal grid staining 

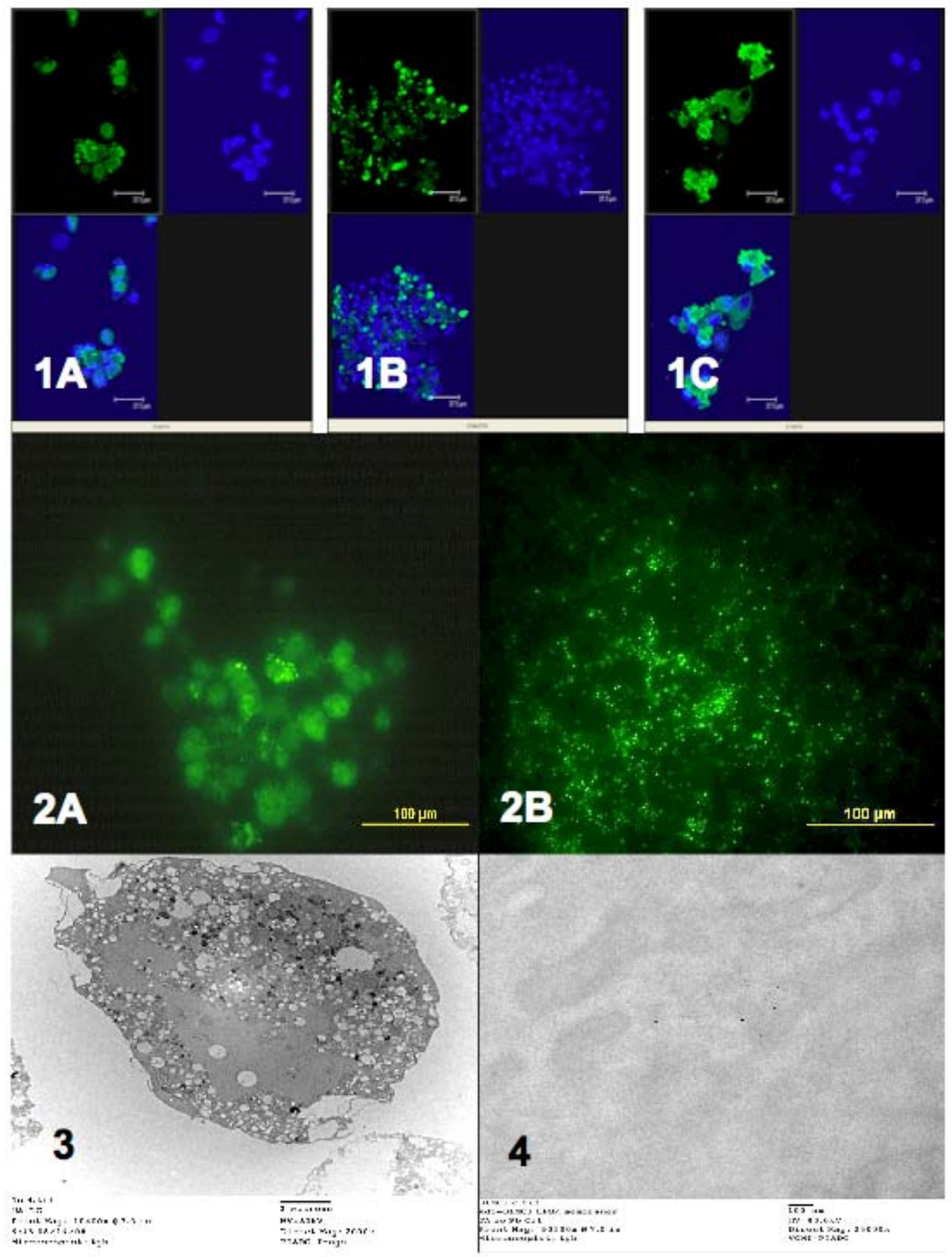